\title{
Violencia de género sobre estudiantes universitarios(as)
}

\author{
Gender violence on college students
}

\begin{abstract}
Francisco José Zamudio-Sánchez / fzamudios@taurus.chapingo.mx orcid.org/0000-0001-8252-9255

Marco Antonio Andrade-Barrera / mandradebs@gmail.com orcid.org/0000-0003-3807-9108

Roxana Ivette Arana-Ovalle / aovalle@colpos.mx orcid.org/0000-0003-2118-2029
\end{abstract}

Arturo A. Alvarado-Segura / arturo.segura@correo.chapingo.mx Universidad Autónoma Chapingo, México

\begin{abstract}
We propose an index to measure gender violence in university students (women and men). To build this index, a questionnaire about violence is applied to a representative sample of students. Then, the information is organized through an ecological model and the result is integrated in an index through the analytic hierarchy process. The proposed index was exemplified for students in the Chapingo Autonomous University (UACh). In the UACh, both women and men showed similar grades of direct violence, but women exhibited more social and emotional consequences. The proposed index allows us: 1) to identify factors that influence the reproduction of violence, 2) to incorporate opinions of experts, 3) to get information of multiple factors and perceptions that people have about violence. Finally, this index can be extended to evaluate others social issues.
\end{abstract}

Key words: aggression, school environment, stereotypes, gender studies, young.

Resumen: Proponemos un índice para medir violencia de género entre hombres y mujeres universitarios(as). Para construir dicho índice se aplicó un cuestionario sobre violencia a una muestra representativa de estudiantes. Posteriormente, se organizó la información mediante un modelo ecológico, y el resultado se integró en un índice vía el proceso jerárquico analítico. El índice propuesto se ejemplificó con datos de alumnos de la Universidad Autónoma Chapingo (UACh). En ésta, tanto hombres como mujeres reportaron grados similares de violencia directa, aunque las mujeres señalaron más consecuencias emocionales y sociales. El índice propuesto permite: 1) identificar los factores que influyen en la reproducción de la violencia, 2) incorporar la opinión de los expertos, 3) obtener información de múltiples factores y percepciones que las personas tienen sobre el fenómeno. Finalmente, este índice puede extenderse para evaluar otros fenómenos sociales.

Palabras clave: agresión, ambiente escolar, estereotipos, estudios de género, jóvenes. 


\section{Introducción ${ }^{1}$}

La violencia es una de las mayores amenazas de salud pública en el mundo. Particularmente, la violencia de género concentra la mayor parte de los tipos de violencia, y la de hombres hacia mujeres, niños(as) y otros hombres son los casos más comunes (Ferguson et al., 2005). Por eso, el estudio de la violencia de género es relevante, sobre todo en las universidades, que tienen un papel fundamental en la formación y difusión de ideologías (Castells, 2001). Entre dichas ideologías se encuentran las reglas que subordinan lo femenino a lo masculino; es decir, un tipo de violencia estructural donde los hombres se ubican en una posición superior (Confortini, 2006: 340), manteniendo con ello las asimetrías de poder y la violencia de género.

Desde 1995, en México se han impulsado diversas políticas públicas para atender la violencia contra las mujeres. Entre ellas podemos mencionar el Programa Nacional de la Mujer, la creación del Instituto Nacional de las Mujeres y de la Fiscalía Especial para los Delitos de Violencia contra las Mujeres y Trata de Personas, así como la Ley General de Acceso a las Mujeres a una Vida Libre de Violencia, entre otras (Centro de Estudios para el Adelanto de las Mujeres y la Equidad de Género, 2012). También podemos mencionar la reforma al artículo $1^{\circ}$ constitucional, en el cual queda prohibido cualquier tipo de discriminación en razón del género.

En las instituciones de educación superior también se han hecho esfuerzos para prevenir y erradicar la violencia de género. Entre éstos podemos mencionar: el violentómetro, diseñado en el Instituto Politécnico Nacional, y las estrategias y propuestas del Programa Universitario de Estudios de Género (PUEG) de la Universidad Nacional Autónoma de México; todas ellas a favor de la equidad, promoviendo, elaborando y coordinando diversas investigaciones. En esta actividad constante, también ha contribuido la Asociación Nacional de Universidades e Instituciones de Educación Superior (Ordorika, 2015). En 2009, se llevó a cabo la declaratoria de la "Reunión Nacional de Universidades Públicas. Caminos para la Equidad de Género en las Instituciones de Educación Superior", en la cual se promueve la igualdad de oportunidades entre las mujeres y los hombres que integran las comunidades universitarias (Red Nacional de Equidad de Género, 2009).

1 Agradecemos al Fondo Sectorial Mexicano SEP/UPEPE/SES/Conacyt el apoyo y financiamiento para la elaboración de este trabajo y a la Universidad Autónoma Chapingo por las facilidades otorgadas. 
En cuanto a las investigaciones relacionadas con la violencia de género enfocadas a estudiantes universitarios (hombres y mujeres), resalta el estudio internacional sobre violencia en la pareja (International Dating Violence Study), en el cual se encuestó a estudiantes universitarios de 32 naciones. Los datos recolectados en dicho estudio han sido insumo de varias investigaciones, en las cuales se observan porcentajes altos de violencia física en la pareja. Aunque no se han encontrado diferencias significativas entre hombres y mujeres, la severidad de los daños que ellos ocasionan a las mujeres generalmente es mayor (Chan et al., 2008; Chan y Straus, 2008; Hines y Straus, 2007; Rebellón et al., 2008).

En los trabajos realizados en México destacan: el estudio sobre Disciplina, Violencia y Consumo de Sustancias Nocivas a la Salud en Escuelas Primarias y Secundarias de México (EDVC) y las encuestas de Exclusión, Intolerancia y Violencia (EIV) (Subsecretaría de Educación Media Superior, 2012). Entre los resultados más relevantes se señala que los hombres participan y son víctimas de violencia en mayor medida que las mujeres, además de existir altos porcentajes de discriminación e intolerancia con personas de diferente color de piel, religión, condición socioeconómica y preferencia sexual (Mingo, 2010; Tapia, 2010; Ávila, 2009; Szekely, 2008; Muñoz, 2008).

La UACh es un espacio donde concurren estudiantes hombres y mujeres de todos los estados de la República Mexicana y ofrece un sistema de becas que permite a los alumnos vivir en un internado o alojarse en departamentos cercanos a la universidad; de modo que el $87 \%$ de ellos(as) viven alejados(as) de su familia. Algunos estudios han sugerido que esta situación sui géneris de la UACh propicia algunas manifestaciones de violencia de género (Vázquez y Castro, 2008 y 2009; Castro y Vázquez, 2008; Montaño et al., 2008; Spitzer, 2002).

En el presente trabajo se propone un índice que puede medir el grado de violencia al cual está sometido un individuo, hombres o mujeres, o incluso todos los integrantes de la Universidad. Este índice permite determinar los factores que más influyen en la generación de la violencia en estos grupos, identificando las manifestaciones de violencia de género que sufre el alumnado de la UACh.

Bajo la hipótesis de que este tipo de violencia está sumamente enraizado en las desigualdades de poder y la asimetría social entre hombres y mujeres (Expósito, 2011; United Nations High Commissioner for Refugees [UNHCR], 2003), y considerando que la UACh ofrece condiciones equitativas a su alumnado, independientemente del sexo, entonces las diferencias capturadas por el índice propuesto sobre los niveles de violencia que sufren hombres y 
mujeres podrán ser atribuidas al género. Cuando se colectó la información para ejemplificar el índice de violencia propuesto en este trabajo, la matrícula consistía en 7,244 estudiantes: 4,368 hombres y 2,876 mujeres. De la matrícula total, 1,306 mujeres y 1,955 hombres estaban cursando la preparatoria, mientras que a nivel licenciatura había 1,570 mujeres y 2,413 hombres.

Con la progresión de los estudios sobre violencia, se ha llegado a descubrir su carácter polifacético y su ubicuidad en diversas escalas (micro, meso, macro o mega) y ámbitos (individuos, familias, grupos, instituciones, civilizaciones) (Jiménez-Bautista, 2012: 18). Por esta razón se decidió proponer un índice que capturara un amplio número de facetas, escalas y ámbitos de la violencia. Para lograrlo, se recurrió al modelo ecológico, propuesto inicialmente por Bronfenbrenner (1979) para estudiar el desarrollo humano, aunque posteriormente otros investigadores lo han ajustado para estudiar la violencia, analizando las interacciones entre factores personales, situacionales y socioculturales (Heise, 1998). Este enfoque ecológico evita recortar y aislar a la persona de su entorno, permitiendo así un punto de vista más amplio sobre el problema (Corsi, 1994).

Para Galtung (1981: 84), un punto de partida y elemento básico de la violencia es la destrucción; y en un nivel más alto de abstracción, la violencia se presenta en cualquier cosa evitable que impida la autorrealización humana. En este sentido, Galtung plantea lo que se conoce como el triángulo de violencia, cuyas esquinas son la violencia cultural, estructural y personal (Confortini, 2006:340). En la violencia directa encontramos manifestaciones verbales, psicológicas y físicas. Por su parte, la violencia estructural se produce a través de mediaciones institucionales o estructurales. Ejemplos de esta violencia son la pobreza y la represión política. Finalmente, la violencia cultural se manifiesta mediante las facetas culturales que apoyan o justifican las realidades y prácticas de violencia (Jiménez-Bautista, 2012).

Para efectos de este estudio se consideró como violencia a "todo acto intencional, que puede ser único o recurrente y cíclico, dirigido a dominar, controlar, agredir o lastimar a otra persona" y que casi siempre es ejercida por las personas de mayor jerarquía (Instituto Nacional de las Mujeres, 2008: 11). Adicionalmente, para que un acto sea considerado violento debe poseer tres características: desorden, avasallamiento y daño. El término violencia de género se distingue de la violencia común porque se dirige a individuos o grupos de individuos en función de su género.

Se encontró que los elementos principales que impulsan la reproducción de la violencia en la UACh son: el abuso de poder, la falta de aplicación 
de las normas, la impunidad y la corrupción. Aunque tanto hombres como mujeres pueden ser víctimas de tales actos, la mayor parte de las agresiones son perpetradas por hombres. En cuanto a las secuelas que genera la violencia, los resultados sugieren que las mujeres son más susceptibles a las consecuencias emocionales y sociales, producto de la violencia sufrida.

\section{Metodología}

El índice de violencia de género entre estudiantes universitarios que se propone en este trabajo fue ejemplificado en la comunidad estudiantil de la UACh, en México. Hombres y mujeres de nivel preparatoria y licenciatura constituyeron la población de estudio. Se adecuaron las ideas principales del modelo ecológico (Corsi, 1994), para delimitar los sistemas de análisis y guiar la construcción de un instrumento para la recolección de los datos.

Los conceptos de violencia se operacionalizaron mediante una adaptación del proceso jerárquico analítico (Saaty, 1986) y la construcción de combinaciones lineales que permiten obtener índices para los distintos niveles y subniveles del modelo ecológico, para el cálculo del índice general, tanto para hombres como para mujeres.

\section{Muestra}

Se planteó un diseño de muestreo estratificado de cuatro estratos: mujeres estudiantes de preparatoria (62 encuestas), hombres de preparatoria (37), mujeres de licenciatura (57) y hombres de licenciatura (51). En total, se encuestaron 119 mujeres y 88 hombres. El rango de edades del alumnado encuestado fue de 14 a 31 años; aproximadamente la mitad tenía entre 18 y 21 años. En cuanto al lugar de procedencia, 61\% eran de las zonas centro-sur y sureste, aunque la muestra incluyó estudiantes de las ocho regiones geográficas del país. También es importante mencionar que 15\% de los alumnos encuestados eran indígenas. Respecto al estado civil, el 92\% eran solteros, 3\% estaban en unión libre o casados, y el resto no respondió.

\section{Instrumento de recolección de datos}

El instrumento de muestreo incluyó 35 preguntas, conformadas por baterías de opciones. Algunas preguntas provinieron de la Encuesta Nacional de Violencia en las Relaciones de Noviazgo 2007 (INEGI, 2007) y de la Encuesta 
Nacional sobre la Dinámica de las Relaciones en los Hogares (ENDIREH) (INEGI, 2006), las cuales fueron adaptadas a la situación de la UACh, mientras que otras fueron incluidas por el equipo de investigación. Los cuestionarios fueron autoadministrados y totalmente anónimos, con la intención de garantizar la confidencialidad de la información.

Para obtener la del sistema individual, se incluyeron preguntas relacionadas con la información personal del entrevistado, entre las que se encuentran aquellas sobre sus hábitos de consumo de alcohol o drogas, el número de parejas y la forma de relacionarse con ellas, la edad a la que tuvo su primera relación sexual, así como la violencia presenciada en la niñez.

En el microsistema, se incluyeron preguntas para caracterizar las experiencias de violencia con familiares cercanos a la persona, tales como el padre y la madre, hermanos(as), tíos(as), abuelos(as), etcétera. También se tomó información acerca de la estabilidad en sus relaciones de pareja y las decisiones que toman en sus relaciones formales, es decir, parejas casadas, en unión libre por más de cinco años o con hijos(as).

En el mesosistema se reunieron variables sobre violencia dentro y en los alrededores de la UACh. Las preguntas intentaron capturar diversas manifestaciones de violencia física, económica, sexual y psicológica, así como los lugares donde ocurre, su frecuencia, los perpetradores de tal violencia (profesores(as), compañeros(as) de clases, personal administrativo, novio(a), etcétera), las lesiones físicas producidas por violencia y la ayuda proporcionada a las víctimas (primeros auxilios, atención médica, psicológica y legal).

Finalmente, en el macrosistema las preguntas pretendieron capturar información sobre las diferentes creencias y estereotipos que legitiman y reproducen la violencia. También, se indagó en cuáles otros lugares fuera de la UACh el alumnado sufre violencia, así como las opiniones que tiene sobre las actitudes, las prácticas y los factores que producen y reproducen violencia en la UACh. Adicionalmente, se solicitaron propuestas que ellos(as) consideran adecuadas para mitigar este problema.

\section{Construcción del indice}

El índice de violencia se construyó siguiendo una modificación del proceso jerárquico analítico, una teoría de medición propuesta por Thomas L. Saaty (1986) en 1980, la cual consiste en un procedimiento sistemático que divide un problema en pequeñas partes que son comparadas por pares para generar prioridades entre ellas. 
En nuestro caso, el problema por medir fue la violencia a la cual ha estado sometido el alumnado de la UACh. Las partes del problema son los elementos considerados en el modelo ecológico. De esta manera, los sistemas (individual, microsistema, mesosistema y macrosistema) fueron la primera partición. Cada sistema se desagregó en variables y éstas, a su vez, en preguntas que contenían varias opciones (véase Figura 1 ). ${ }^{2}$

Antes de calcular el índice de violencia por pregunta, se generó un ordenamiento, estableciendo la gravedad de la violencia de cada una de las opciones de todas las preguntas del instrumento. Para realizar esto, se tuvieron en cuenta dos criterios:

El primero fue adoptar una escala del 1 al 7 para hacer comparaciones entre las partes, fijando el valor 1 sobre aquellas consideradas de menor gravedad (prioridad) y asignando valores mayores a 1 a las consideradas más graves. Por ejemplo, si se veía que una de las partes era tres veces más grave que las de valor 1 , entonces a ésta se le asignaba el número 3.

Este proceso requiere que personas expertas en la temática emitan los juicios para establecer las prioridades, para que el ordenamiento resultante sea congruente con lo que se sabe sobre el fenómeno. Las calificaciones de gravedad de la violencia para cada opción en el proceso jerárquico analítico fueron realizadas por investigadores enfocados al género y especialistas en estadística.

El segundo criterio ayudó a determinar qué tan grave era una parte respecto a otra (entre opciones de preguntas, entre preguntas, entre variables y entre sistemas). En general, se consideró que un acto de violencia proveniente de alguien más cercano al individuo (familia) tiene repercusiones más graves que el proveniente de alguien más lejano. Por ejemplo, se consideró más grave un acto de violencia perpetrado por la pareja (más cercano) que por un desconocido. Asimismo, se observó como más grave sufrir un acto de violencia en el hogar, que en la comunidad. Otras reglas particulares para comparar la magnitud de las situaciones consideraron los riesgos que generan éstas. Por ejemplo, se consideró más grave el consumo de alcohol o drogas respecto al no consumo.

Una vez determinado esto, se calculó un peso para cada opción de las preguntas, dividiendo la gravedad de la opción entre la suma de todas las gravedades asociadas a las opciones de la pregunta en cuestión. De este modo, la construcción del índice general de violencia se puede explicar en cuatro pasos, como se describe en seguida. Los cálculos en cada paso se hicieron para todos los cuestionarios.

2 Todas las tablas y gráficas se encuentran en el Anexo, al final del presente documento. 
En el paso 1, se calculó el índice de violencia de una pregunta particular utilizando la combinación lineal de los pesos y las frecuencias (esto es, la sumatoria del producto de los pesos por las frecuencias) que los(as) participantes respondieron en el cuestionario.

\section{ÍndiceViolenciaPregunta_ $i=\sum($ PesoOpción $j)($ FrecuenciaOpción $j$ )}

Aunque esta agregación puede ser mayor a 1, se decidió tomar el mínimo entre 1 y la combinación lineal, considerando que si alguien alcanzaba el valor 1, entonces esta persona se encontraría en una situación crítica de violencia; por el contrario, valores cercanos a 0 en el índice indican situaciones de violencia leve o casi nula. Cabe destacar que se calcularon tantos índices como preguntas tuvo el cuestionario.

En el paso 2, se construyó análogamente un índice para cada variable. En este caso, se juzgó la prioridad de las preguntas relacionadas con una variable. Una vez calculado el peso de cada pregunta de acuerdo con su prioridad, se realizó la combinación lineal del índice de violencia de la pregunta con su respectivo peso.

\section{ÍndiceViolenciaVariable_k $k=\sum$ (PesoPregunta_i)(ÍndicePregunta_i)}

A partir de los índices de violencia de cada variable calculados para cada cuestionario, se obtuvo el promedio para hombres, para mujeres y el general.

En el paso 3, se construyeron índices de violencia para cada sistema, como una combinación lineal del peso de cada variable que forma el sistema y el índice de la variable obtenido en el paso 2.

$$
\text { ÍndiceViolenciaSistema_l }=\Sigma(\text { PesoVariable_ } k)(\text { ÍndiceVariable_ } k)
$$

A partir de los índices de violencia de cada sistema calculados para cada cuestionario, se obtuvo el promedio para hombres, para mujeres y el general.

Finalmente, en el paso 4, se obtuvo el índice general de violencia que consistió en una combinación lineal de los pesos de cada sistema y los índices respectivos. De esta manera, toda la información recolectada en el cuestionario se integró de forma anidada en el índice general. Este índice puede expresarse como:

ÍndiceGeneralViolencia $=\sum($ PesoSistema_l $)$ (ÍndiceSistema_l $)$

Con base en los índices de violencia general de cada cuestionario, se obtuvo el promedio para hombres, para mujeres y el general. 


\section{Resultados}

En general, la violencia que experimentaron las mujeres (índice de 0.2084) fue 5.6 puntos porcentuales mayor que la de los hombres (índice 0.1974). Aunque esta diferencia no es estadísticamente significativa, con un $10 \%$ de error y una confianza del 90\%, algunas variables, como Estereotipos y Consecuencias de la violencia, sí mostraron diferencias significativas entre ambos sexos. Estas variables se encuentran entre las que tuvieron mayor aportación al valor del índice general de violencia.

La siguiente descripción es útil para dimensionar mejor los resultados; los ejemplos que se mencionan fueron obtenidos de la información recolectada en la muestra y representan aproximadamente algunos valores particulares del índice:

- A una persona con un valor de aproximadamente 0.05 le han puesto apodos, ha sufrido violencia física en su familia, se ha sentido violentada en el transporte público, y, por lo general, en su relación de pareja sólo ella es quien toma las decisiones.

- Alguien con un valor cercano a 0.20 ha sido empujado e insultado varias veces, le han puesto apodos, sus compañeros de clases lo han agredido, sus padres lo han violentado de manera física y psicológica, tiene estereotipos en los que subordina a las mujeres, consume bebidas embriagantes y sólo él toma las decisiones de pareja.

- Una persona que presenta un valor de 0.40 ha sido pateada y la han golpeado con la mano, su pareja la ha chantajeado económicamente, la han acosado sexualmente, la han menospreciado, siente miedo por la violencia que ha sufrido y ha tenido moretones o rasguños debido a esa violencia. Además, se siente triste y deprimida, ha tenido problemas por consumir alcohol y ha experimentado violencia en su familia con sus padres y hermanos(as).

Como se observa, un incremento en el valor del índice se asocia con un incremento en la violencia y en la gravedad de ésta. En las tablas 1-4 se presentan los grados de violencia encontrados en los componentes de cada uno de los sistemas. También se muestra la contribución porcentual que tuvo cada componente, en la conformación de la medida del elemento superior en la jerarquía. Por ejemplo, los porcentajes de las variables en la tabla del macro-sistema representan la contribución promedio que tuvo cada una de esas variables en el índice promedio del sistema. 
De forma similar, los porcentajes de las preguntas equivalen a la aportación promedio que tuvieron éstas en su variable correspondiente. En las tablas 1-4 se presentan todas las componentes del índice desde el nivel de pregunta, con la finalidad de servir como referencia para investigaciones futuras. En este trabajo se discuten las componentes que muestran los valores más altos por su relevancia para explicar los niveles de violencia.

En términos generales, el macrosistema fue el que tuvo la mayor aportación al grado total de violencia, con $49.68 \%$ (véase Tabla 1), mientras los sistemas meso e individual aportaron de manera conjunta (prácticamente en partes iguales) el $41 \%$ del total de la violencia contabilizada (véanse Tabla 2 y Tabla 4), y finalmente, el microsistema fue el que tuvo la menor aportación, con un $9.47 \%$ (véase Tabla 3 ).

En cuanto a la conformación de la medida promedio dentro del macrosistema (véase Tabla 1), de acuerdo con la teoría de Galtung, podemos notar dos tipos de violencia. La variable Reproducción de la violencia dentro de la $U A C h$ fue la que más aportó (54.25\%), al indicar un tipo de violencia estructural. $\mathrm{Al}$ menos el $61 \%$ de los(as) estudiantes mencionan la existencia de delitos evidentes dentro de la UACh que no son sancionados, que las instancias del orden y disciplina no aplican la normatividad (62\%) y que hay abuso de poder (70\%).

Por su parte, la segunda variable que representa más violencia fue Estereotipos de género y de violencia, al señalar un tipo de violencia cultural. En particular la pregunta 31 -en la cual se capturaron algunos estereotipos de género-, el valor del índice en los hombres fue ligeramente superior al de las mujeres, aunque sin diferencia significativa. En esta pregunta se encontró cierta oposición de las estudiantes a algunos estereotipos de género, señalada también por otros estudios (Castro y Vázquez, 2008), donde las alumnas reivindican la condición femenina. Por ejemplo, en el enunciado En el noviazgo la autoridad la tiene el hombre, el $96.66 \%$ ( $\pm 2.73 \%)$ de ellas estuvo en desacuerdo y el resto estuvo total o parcialmente de acuerdo.

Dentro del mesosistema (véase Tabla 2), las variables que tuvieron la mayor aportación al promedio de la medida de violencia fueron Agresores y Tipos de violencia en la UACh. En especial, la variable Agresores señaló uno de los resultados más importantes en la investigación. Por un lado, el $62.60 \%( \pm 8.67 \%)$ de los estudiantes y el $60.59 \%( \pm 7.43 \%)$ de las estudiantes indicaron que han sido agredidos(as) por hombres dentro de la UACh. Por otro lado, $24.93 \%( \pm 7.75 \%)$ de los estudiantes y $29.49 \%( \pm 6.98 \%)$ de las estudiantes indican que han sufrido agresiones de mujeres dentro de la UACh. 
La segunda variable más importante en el mesosistema fue Tipos de violencia en la $U A C h$, la cual incluye la violencia directa del triángulo de Galtung que observa Confortini (2006: 349). En ella la violencia psicológica fue la más señalada $(77.82 \%, \pm 2.89 \%)$, seguida de la física $(45.89 \%$, $\pm 3.48 \%)$, la económica $(45.48 \%, \pm 3.49 \%)$ y la sexual $(27.16 \%, \pm 3.08 \%)$. En cuanto al grado de estudios, el orden se mantiene: en nivel licenciatura $80.6 \%$ experimentó violencia psicológica, mientras que en nivel preparatoria fue un $74.7 \%$. Los valores para los otros tipos de violencia fueron: violencia física ( $45 \%$ licenciatura y $46 \%$ preparatoria), violencia económica (48\% y 42\%, respectivamente) y violencia sexual ( $32 \%$ y $21 \%$, respectivamente).

Respecto a este último tipo de violencia, Castro y Vázquez (2008: 605) encontraron que en las estudiantes de la UACh existe un acoso sistemático, de "baja intensidad" (en tanto que no constituye agresiones físicas directas). En nuestro estudio, dentro de los actos de violencia sexual que experimenta el alumnado, el acoso sexual fue el más mencionado (23.69\% de las mujeres y $10.38 \%$ de los hombres).

En el microsistema (véase Tabla 3), dos variables fueron las que más aportaron: Violencia de origen (52.33\%) y Conducta en su entorno (38.86\%). El tipo de violencia más señalado, del que fueron objeto en sus familias de origen fue el psicológico, con diferencia significativa entre hombres $(29.45 \%)$ y mujeres (42.14\%). En cuanto a la variable Conducta en su entorno, que proporciona información sobre la toma de decisiones en pareja, el valor promedio de este índice para las mujeres fue $29 \%$ mayor que para los hombres, con valores de 0.1253 y 0.0968 , respectivamente.

Finalmente, en el sistema individual (véase Tabla 4), la variable Consecuencias emocionales y/o sociales de la violencia fue determinante; el $64.27 \%$ de la medida promedio en el sistema se debió a dicha variable. Las mujeres fueron quienes reportaron mayores consecuencias de la violencia, pues el promedio del índice se situó 34\% por arriba del valor de los hombres.

En el sistema individual, la variable Hábitos de consumo de alcohol y/o drogas también tuvo una diferencia significativa entre sexos, colocando en una peor situación a los hombres. Este resultado está en concordancia con uno de los discursos sobre masculinidad, identificado por Vázquez y Castro (2009: 707), presente en estudiantes varones de la UACh; hablamos de la necesidad de tomar riesgos durante la juventud para "hacerse hombres", particularmente el consumo de alcohol, cigarro y drogas.

En el caso de los hombres, $50.48 \%( \pm 8.68 \%)$ de ellos afirmó que toma alcohol, y en el caso de las mujeres, el porcentaje fue $33.78 \%$ ( $\pm 7.04 \%)$. Aunque el consumo de alcohol en mujeres no es bajo, la frecuencia con la que lo hacen ellas es, en general, menor que en los hombres. 


\section{Discusión}

La construcción y aplicación constante de medidas de violencia como las que se han propuesto en este documento resultan de mucho valor. Dadas las múltiples recomendaciones, programas y leyes, cuyo objetivo es prevenir y erradicar la violencia, las instituciones, incluyendo las universidades, usualmente se ven involucradas en actividades que buscan el mismo objetivo. Por eso resulta pertinente contar con herramientas que puedan medir el cambio en los comportamientos violentos o en factores específicos de la violencia.

El índice propuesto en este trabajo no pretende ser en extremo preciso; sin embargo, por la naturaleza de su construcción, es acumulativo. Los actos de violencia se agregan sin necesidad de contar con una unidad de medida (es más violento haber sido empujado y pateado en comparación con haber sido sólo empujado). De aquí que los valores del índice sean ordinales y, por lo tanto, permitan evaluar si hay mayor o menor violencia.

Los factores que tuvieron mayor contribución al problema de violencia pertenecen al macrosistema. Esto puede explicarse debido a que la sociedad impone al individuo sus costumbres y creencias. Dado que la sociedad mexicana tiene una estructura patriarcal, las relaciones de poder entre hombres y mujeres provocan desigualdades entre sexos, lo cual, en muchos casos, genera situaciones de violencia (Cagigas, 2000).

Un reflejo de esta estructura patriarcal se manifiesta en los estereotipos de género, que en general tienden a mantener la dominación masculina. En la búsqueda de la equidad de género, los resultados señalan que las estudiantes de la UACh muestran oposición a estos estereotipos, que es más fuerte comparada con otros estudios realizados en mujeres y que coincide con trabajos previos como el de Castro y Vázquez (2008). No obstante, la diferencia observada entre hombres y mujeres en la variable Conducta en su entorno sugiere cierta aceptación de roles de género en la pareja por parte de las estudiantes, pues consideran que varias de las decisiones de pareja, como las asociadas con las relaciones sexuales y los hijos, deben ser tomadas exclusivamente por las mujeres.

La presencia de estereotipos en los hombres resultó similar a las mujeres. Otras investigaciones como la de Montaño et al. (2008) cuestionan e incluso se sorprenden con este tipo de resultados, donde los hombres parecen estar en pro del empoderamiento de las mujeres, cuando menos tanto como las mujeres mismas. Las autoras piensan que los varones no contestan con la verdad pues no reconocen su violencia, les da miedo o sienten vergüenza. Aunque es difícil estar seguros de tal conclusión, otros resultados podrían estar asociados con esa falta de transparencia en los hombres. 
Por ejemplo, Vázquez y Castro (2009: 715) llaman a esto una coraza de masculinidad. En su estudio, identificaron la masculinidad en los estudiantes de la UACh como una camisa de fuerza en la que se ven atrapados los hombres, una armadura, que al ser abierta con una llave como el alcohol, permite emerger conductas como el llanto, los tocamientos físicos entre varones y las reiteradas declaraciones de afecto y amistad.

En nuestro estudio, una posible manifestación de esta coraza se encontró en la variable Consecuencias de la violencia. Muchos hombres parecen ser inmunes a las consecuencias emocionales o sociales de la violencia, o, al menos, la cultura no les permite mostrar debilidad. No se detectaron diferencias significativas en la cantidad y gravedad de hechos violentos que sufren hombres y mujeres; sin embargo, la medida de violencia captó un contraste que se presenta entre ambos sexos, pues las mujeres tuvieron un valor $34 \%$ mayor que los hombres en la variable Consecuencias de la violencia.

Aunado a la violencia sufrida en la familia de origen (que resultó equivalente entre hombres y mujeres), el proceso de domesticación, ${ }^{3}$ señalado por Castro y Vázquez (2008), se refuerza con el acoso constante que reciben las estudiantes por parte de los hombres. No obstante, también los hombres sufren los estragos de la violencia general provocada por otros hombres. Posiblemente, parte de esta violencia ejercida por hombres se encuentre en esa necesidad de tomar riesgos para "hacerse hombres", tales como el consumo de alcohol. Spitzer (2002) señala que en casos de indisciplina en estudiantes de la UACh, hay algunos que implican la violencia masiva y excesiva bajo los efectos del alcohol.

Por su parte, el caso de mujeres agresoras no es un hecho aislado, pues un alto porcentaje, tanto de hombres como de mujeres, reporta haber sido agredido por mujeres. Esto no es un fenómeno nuevo, otros estudios han mostrado también que el binomio hombre-victimario, mujer-víctima es un esquema que no siempre ocurre (Hernández, 2009).

Aunque una parte del problema podría recaer en las instancias universitarias, otra podría atribuirse a los mismos afectados, quienes no afrontan la injusticia, arguyendo que el gasto de tiempo (o dinero) que implicaría insistir en que se haga justicia puede ser tan grande, que prefieren olvidar el asunto. Cuando la justicia no se ejerce apropiadamente, se crea un desaliento entre las personas para defender sus derechos, y ello abona aún más la impunidad que permea en la sociedad. No obstante, como se mencionó al principio de este documento, desde hace varios años en México se han realizado diversos

3 Castro y Vázquez (2008) identifican la domesticación de las mujeres como un proceso mediante el cual se les somete de diversas maneras a las reglas de la dominación masculina. 
esfuerzos mediante políticas públicas y reformas de ley, que pretenden combatir la violencia y la inequidad de género.

En este sentido, otra posibilidad es el desconocimiento de todas estas actividades en pro de una vida libre de violencia. En trabajos posteriores se podrían incluir reactivos en el instrumento que den cuenta de los niveles de conciencia de los sujetos sobre sus garantías individuales y los diversos programas en favor de las mujeres y las víctimas de algún tipo de violencia.

\section{Conclusiones}

Proponemos un esquema para obtener información de múltiples factores, situaciones, comportamientos y percepciones que las personas tienen y que están asociadas con la violencia a la cual están expuestas. Entre las ventajas de esta propuesta es que puede ser utilizada para evaluar otros fenómenos sociales, como la medición de niveles de inseguridad, felicidad o desarrollo humano. De acuerdo con los resultados obtenidos, el índice de violencia propuesto parece capturar adecuadamente las manifestaciones de violencia, dada la consistencia en sus resultados y en las comparaciones realizadas con otros estudios en Chapingo. Sin embargo, tiene puntos débiles.

Para determinar la gravedad de la violencia siempre se consideró un mismo acto en diversos ámbitos. Por mencionar un ejemplo, se consideró más grave recibir una patada en el hogar en comparación con recibir una patada en la calle. Esta noción de cercanía utilizada para determinar la gravedad puede ser debatible. Por esta razón, en trabajos futuros será necesario hacer otras evaluaciones del índice para asegurar su confiabilidad y validez.

En el análisis de los casos de la UACh, la variable que más aportó al índice fue Reproducción de la violencia, la cual indica que el abuso de poder, la impunidad y la corrupción son elementos que determinan fuertemente el ejercicio de la violencia.

El índice de violencia señaló hallazgos que hubiese sido difícil detectarlos utilizando sólo las estimaciones parciales de las preguntas, como el hecho de que tanto los estudiantes como las estudiantes, están bajo grados de violencia equivalentes, aunque los tipos de violencia, la frecuencia y la intensidad están diferenciados entre sexos. La ventaja de usar un índice de violencia es que en un sólo valor se integra y se representa la información contenida en un gran número de reactivos, aun cuando pudieran perderse algunos elementos. La descomposición que se hace por sistema, variable y por subgrupos poblacionales ayudará a identificar los factores que reproducen la violencia, y, por ende, se podrán realizar estrategias de corrección dirigidas. 
Concluimos que la situación de violencia en la UACh es un problema estructural y que gran parte de él se debe a la falta de sanción de los hechos violentos. No obstante, los resultados de esta investigación sugieren que programas universitarios dirigidos a los agresores, y no sólo a las víctimas, podrían disminuir la cantidad de hechos violentos. También consideramos necesaria la creación de un Comité de Equidad de Género que emprenda acciones y dé seguimiento a la equidad de género dentro de la institución, lo cual podría instrumentarse a través de diagnósticos periódicos, usando índices como el propuesto en este documento.

Finalmente, podemos afirmar que la situación de violencia en la UACh no se asocia preferentemente con el sexo de la víctima y sí con una condición de poder, y aunque el porcentaje de personas agredidas por mujeres no es bajo, los principales agresores son los hombres.

\section{Referencias}

Ávila, Alejandro (2009), "Violencia homofóbica en Hidalgo. Historia de una omisión", en Vargas, Pablo [coord.], Hidalgo. La memoria contra el olvido. Violencia social y de género, México: Academia Hidalguense de Educación y Derechos Humanos.

Bronfrenbrenner, Urie (1979), The ecology of human development: experiments by nature and design, EUA: Harvard University Press.

Cagigas, Ana (2000), "El patriarcado, como origen de la violencia doméstica", en Monte Buciero, núm. 5, España: Ayuntamiento de Santoña.

Castells, Manuel (2001), "Universities as dynamic systems of contradictory functions", en Muller, Johan et al. [eds.], Challenges of globalisation, South African debates with Manuel Castells, Sudáfrica: Maskew Miller Longman.

Castro, Roberto y Vázquez, Verónica (2008), "La Universidad como espacio de reproducción de la violencia de género. Un estudio de caso en la Universidad Autónoma Chapingo", en Estudios Sociológicos, vol. 26, núm. 3, México: El Colegio de México.

Centro de Estudios para el Adelanto de las Mujeres y la Equidad de Género (CEAMEG) (2012), Violencia de género en México: Estadísticas, marco jurídico, presupuesto, políticas públicas, México: CEAMEG.

Chan, Ko Ling y Straus, Murray (2008), "Prevalence and correlates of physical assault on dating partners", en The Open Social Science Journal, vol. 1, United Arab Emirates: Bentham Science Publishers.

Chan, Ko Ling et al. (2008), "Prevalence of dating partner violence and suicidal ideation among male and female university students worldwide", en Journal of Midwifery \& Women's Health, vol. 53, núm. 6, EUA: The American College of Nurse-Midwifes. Disponible en: http://dx.doi.org/10.1016/j.jmwh.2008.04.016 [2 de febrero de 2017].

Confortini, Catia (2006), "Galtung, Violence, and Gender: The Case for a Peace Studies/ Feminism Alliance", en Peace \& Change, vol. 31, núm. 3, EUA: Peace History Society and Peace and Justice Studies Association. Disponible en: http://onlinelibrary.wiley. com/doi/10.1111/j.1468-0130.2006.00378.x/abstract [2 de febrero de 2017]. 
Corsi, Jorge (1994), "Una mirada abarcativa sobre el problema de violencia familiar", en Corsi, Jorge [comp.], Violencia familiar. Una mirada interdisciplinaria sobre un grave problema social, Argentina: Paidós.

Expósito, Francisca (2011), "Violencia de género", en Mente y Cerebro, vol. 48, España: Investigación y Ciencia.

Ferguson, Harry et al. (2005), Poniendo fin a la violencia de género, Suecia: Edita Västra Aros.

Galtung, Johan (1981), "The specific contribution of peace research to the study to the study of violence: typologies", en Domenach, Jean-Marie et al. [eds.], Violence and its causes, Francia: Naciones Unidas, Imprimerie des Presses Universitaires de France.

Heise, Lori (1998), "Violence against women; an integrated, ecological framework", en Violence Against Women, vol. 4, núm. 3, EUA: Sage. Disponible en http://dx.doi.org/ $10.1177 \% 2 F 1077801298004003002$ [2 de febrero de 2017].

Hernández, Alfonso (2009), "Reseña de El hombre maltratado por su mujer: una realidad oculta”, en Revista de Estudios de Género. La ventana, vol. 29, México: Universidad de Guadalajara.

Hines, Denise y Straus, Murray (2007), "Binge drinking and violence against dating partners: the mediating effect of antisocial traits and behaviors in a multinational perspective", en Aggressive Behavior, vol. 33, EUA: International Society for Research on Aggression. Disponible en http://onlinelibrary.wiley.com/enhanced/exportCitation/ doi/10.1002/ab.20196 [2 de febrero 2017].

INEGI (Instituto Nacional de Estadística y Geografía) (2006), Encuesta Nacional sobre la Dinámica de las Relaciones en los Hogares 2006. ENDIREH, Informe y marco conceptual, México: INEGI.

INEGI (Instituto Nacional de Estadística y Geografía) (2007), Encuesta Nacional sobre Violencia en el Noviazgo 2007. Informe operativo, México: INEGI.

Inmujeres (Instituto Nacional de las Mujeres) (2008), La violencia en las relaciones de noviazgo-pareja y los riesgos en la salud sexual y reproductiva de las y los jóvenes normalistas del Estado de Veracruz, México: Inmujeres. Disponible en: http://cedoc. inmujeres.gob.mx/lgamvlv/Veracruz/noviazgo_veracruz.pdf [15 de septiembre de 2015].

Jiménez-Bautista, Francisco (2012), “Conocer para comprender la violencia: origen, causas y realidad”, en Convergencia Revista de Ciencias Sociales, núm. 58, México: Universidad Autónoma del Estado de México.

Mingo, Araceli (2010), “Ojos que no ven... Violencia escolar y género”, en Perfiles educativos, vol. 32, núm. 130, México: Universidad Nacional Autónoma de México.

Montaño, María et al. (2008), "Violencia de género en la Universidad Autónoma Chapingo", en Martínez, Rosa, Benito Ramírez y Gustavo Rojo [coords.], Estudios y propuestas para el medio rural (tomo $V$ ), México: Universidad Autónoma Indígena de México y Colegio de Postgraduados, Campus Puebla.

Muñoz, Gustavo (2008), "Violencia escolar en México y en otros países. Comparaciones a partir de los resultados del Instituto Nacional para la Evaluación de la Educación”, en Revista Mexicana de Investigación Educativa, vol. 13, núm. 39, México: Consejo Mexicano de Investigación Educativa.

Ordorika, Imanol (2015), "Equidad de género en la Educación Superior", en Revista de la Educación Superior, vol. XLIV, núm. 174, México: Asociación Nacional de Universidades e Instituciones de Educación Superior. 
Rebellon, Cesar et al. (2008), "Self-control in global perspective: an empirical assessment of Gottfredson and Hirschi's general theory within and across 32 national settings", en European Journal of Criminology, vol. 5, núm. 3, EUA, Inglaterra, India y Singapur: Sage. Disponible en: http://journals.sagepub.com/doi/abs/10.1177/1477370808090836\# [2 de febrero de 2017].

Red Nacional de Equidad de Género (2009), Declaratoria. Reunión Nacionalde Universidades Públicas. Caminos para la Equidad de Género en las Instituciones de Educación Superior, México: Universidad Nacional Autónoma de México. Disponible en: http://equidad. pueg.unam.mx/?q=declaratoria [17 de septiembre de 2016].

Saaty, Thomas (1986), "Axiomatic foundation of the analytic hierarchy process", en Management Science, vol. 32, núm. 7, EUA: The Institute for Operations Research and the Management Sciences.

Spitzer, Terry (2002), "Disciplina, violencia estudiantil y género en la Universidad Autónoma Chapingo”, en Educar, vol. 20, México: Universidad de Guadalajara.

Subsecretaría de Educación Media Superior (SEMS) (2012), Encuestas de exclusión, intolerancia y violencia, México: Secretaría de Educación Pública y SEMS. Disponible en: http://www.sems.gob.mx/es_mx/sems/encuestas_dc [15 de septiembre de 2015].

Székely, Miguel (2008), 1 a Encuesta Nacional Exclusión, Intolerancia y Violencia en escuelas públicas de educación media superior, México: Secretaría de Educación Pública. Disponible en: http://www.catedradh.unesco.unam.mx/BibliotecaV2/Documentos/ Educacion/Informes/SEP_Encuesta_Discriminacion_y_Violencia_2008.pdf [15 de septiembre de 2015].

Tapia, Daniel (2010), "La educación como camino para impulsar la libertad en contextos de violencia e intolerancia”, en Sarmiento, Sergio [coord.], Cuarto concurso de ensayo: Caminos de la libertad. Memoria, México: Fomento Cultural Grupo Salinas.

UNHCR (United Nations High Commissioner for Refugees) (2003), Sexual and Genderbased Violence against Refugees, Returnees and Internally Displaced Persons, Suiza: UNHCR.

Vázquez, Verónica y Castro, Roberto (2008), “¿Mi novio sería capaz de matarme? Violencia en el noviazgo entre adolescentes de la Universidad Autónoma Chapingo”, en Revista Latinoamericana de Ciencias Sociales, Niñez y Juventud, vol. 6, núm. 2, Colombia: Universidad de Manizales y Fundación Centro Internacional de Educación y Desarrollo Humano.

Vázquez, Verónica y Castro, Roberto (2009), “Masculinidad hegemónica, violencia y consumo de alcohol en el medio universitario", en Revista Mexicana de Investigación Educativa, vol. 14, núm. 42, México: Consejo Mexicano de Investigación Educativa.

\section{Anexo}




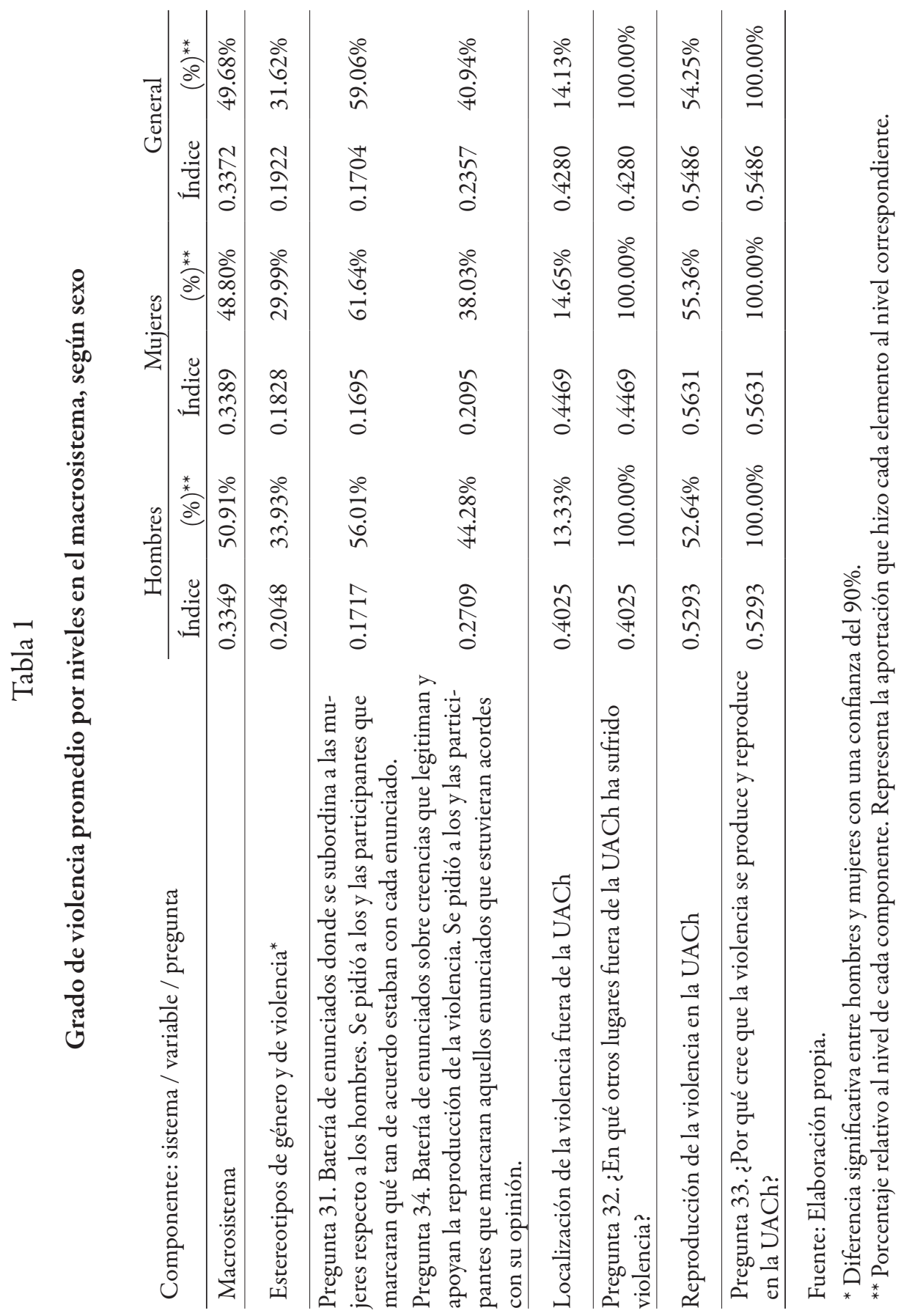




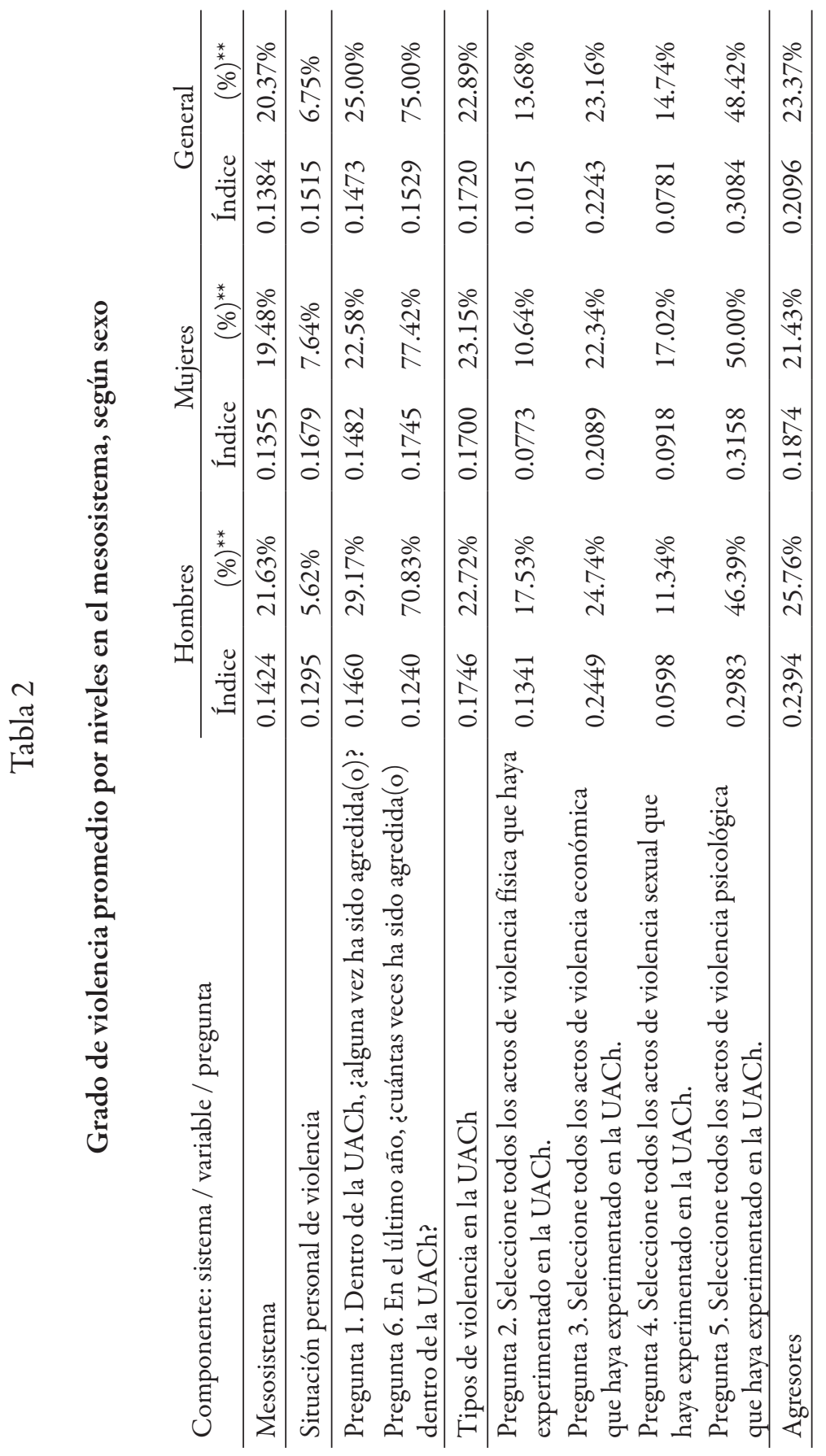




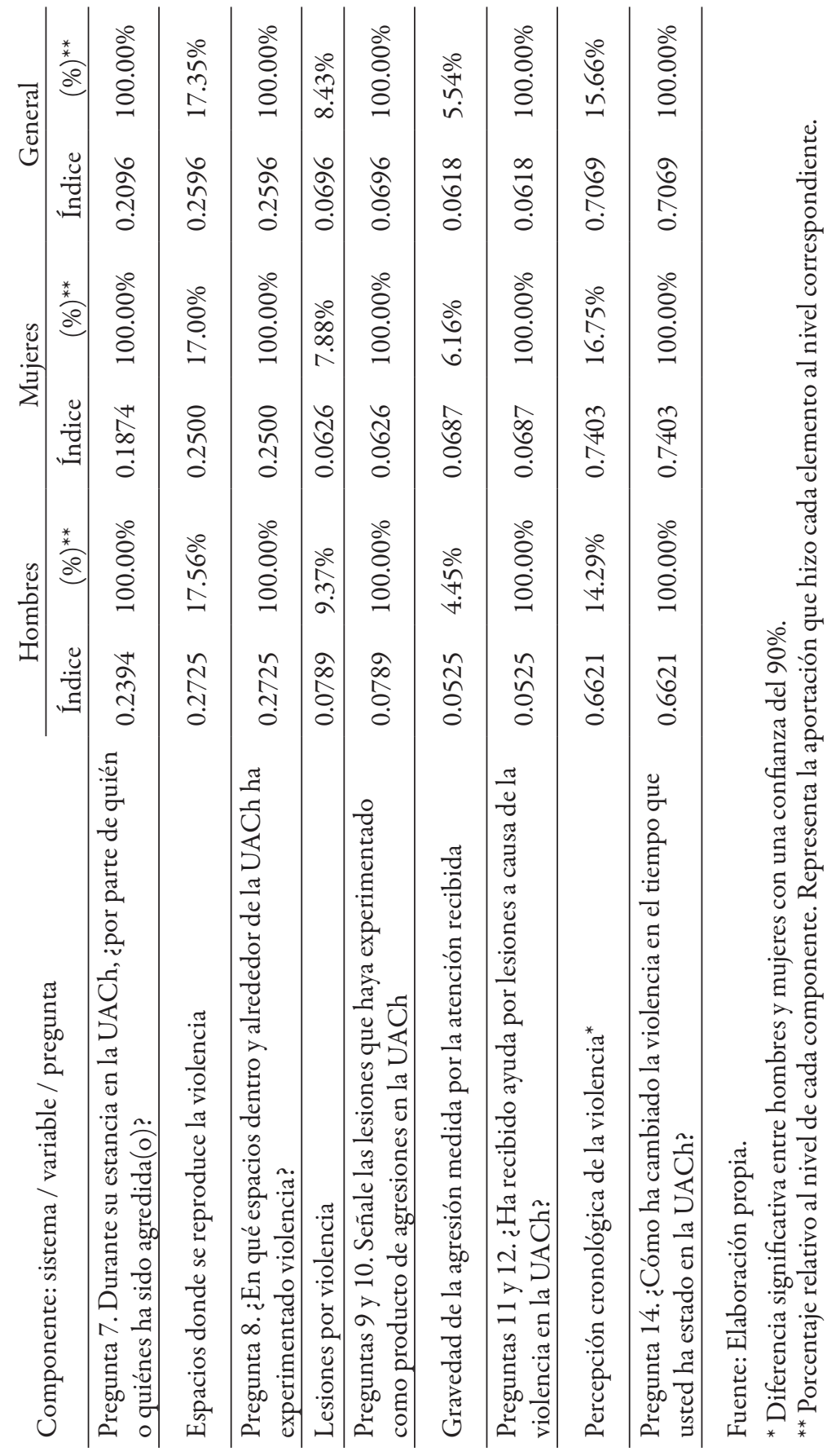




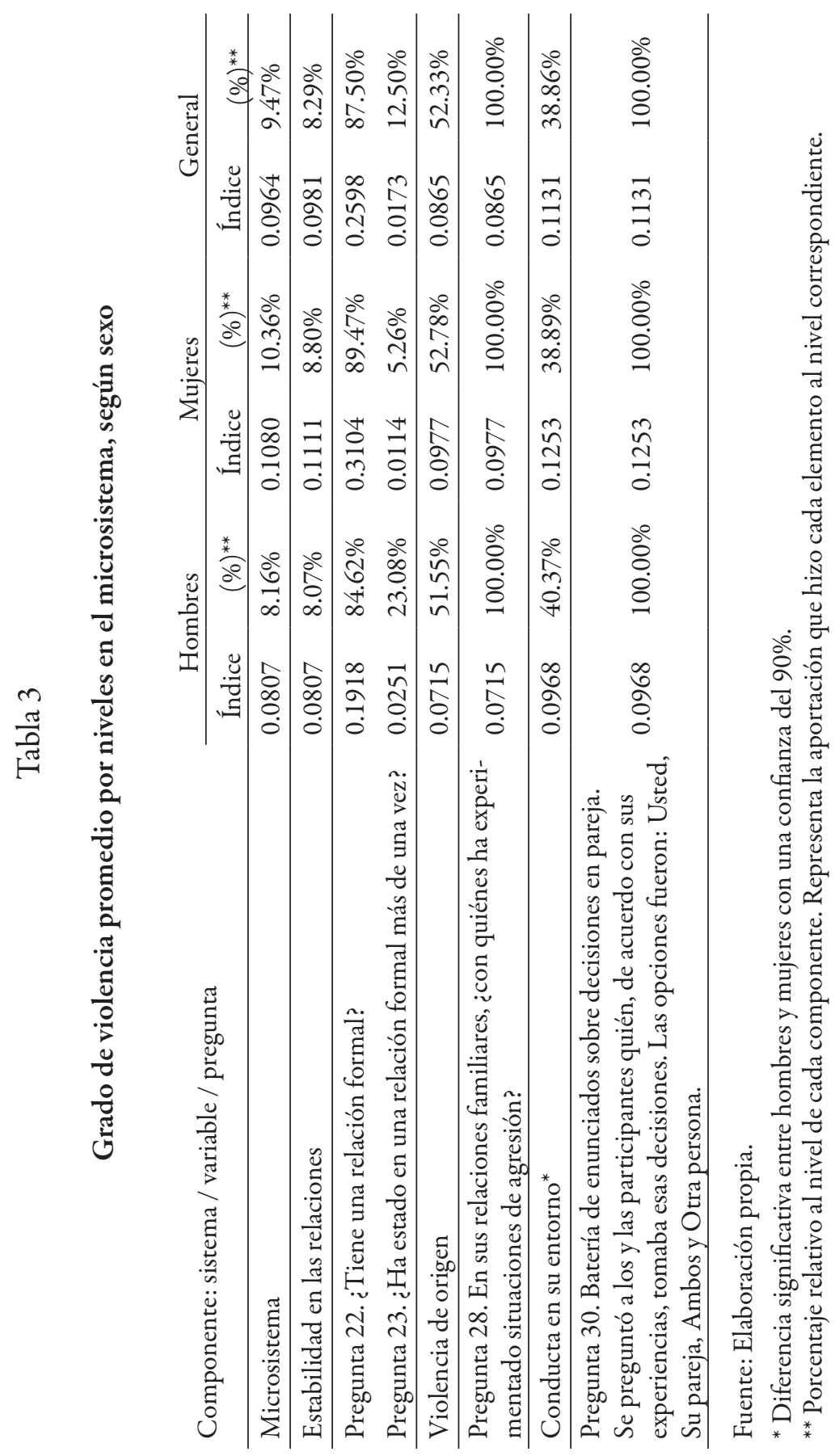




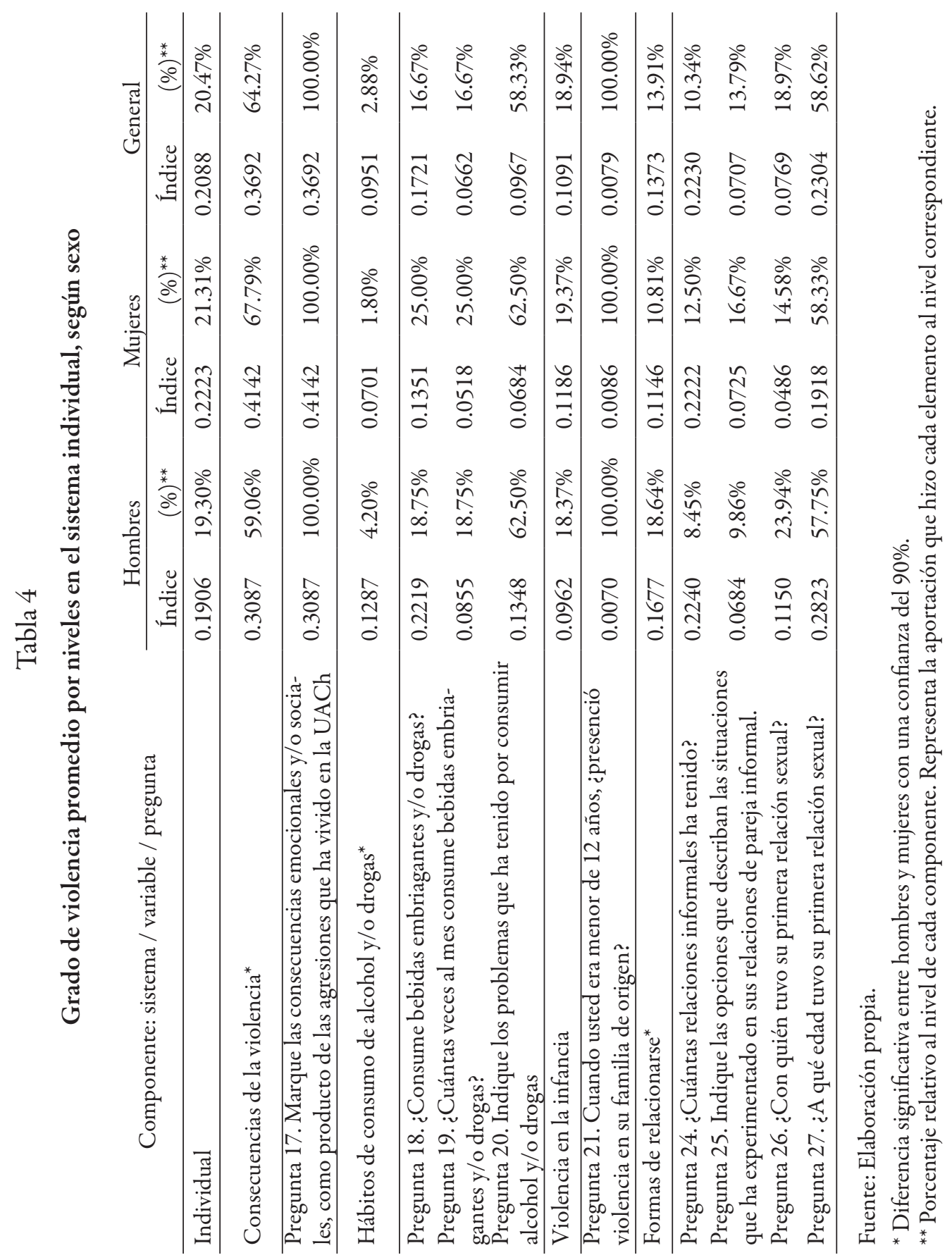



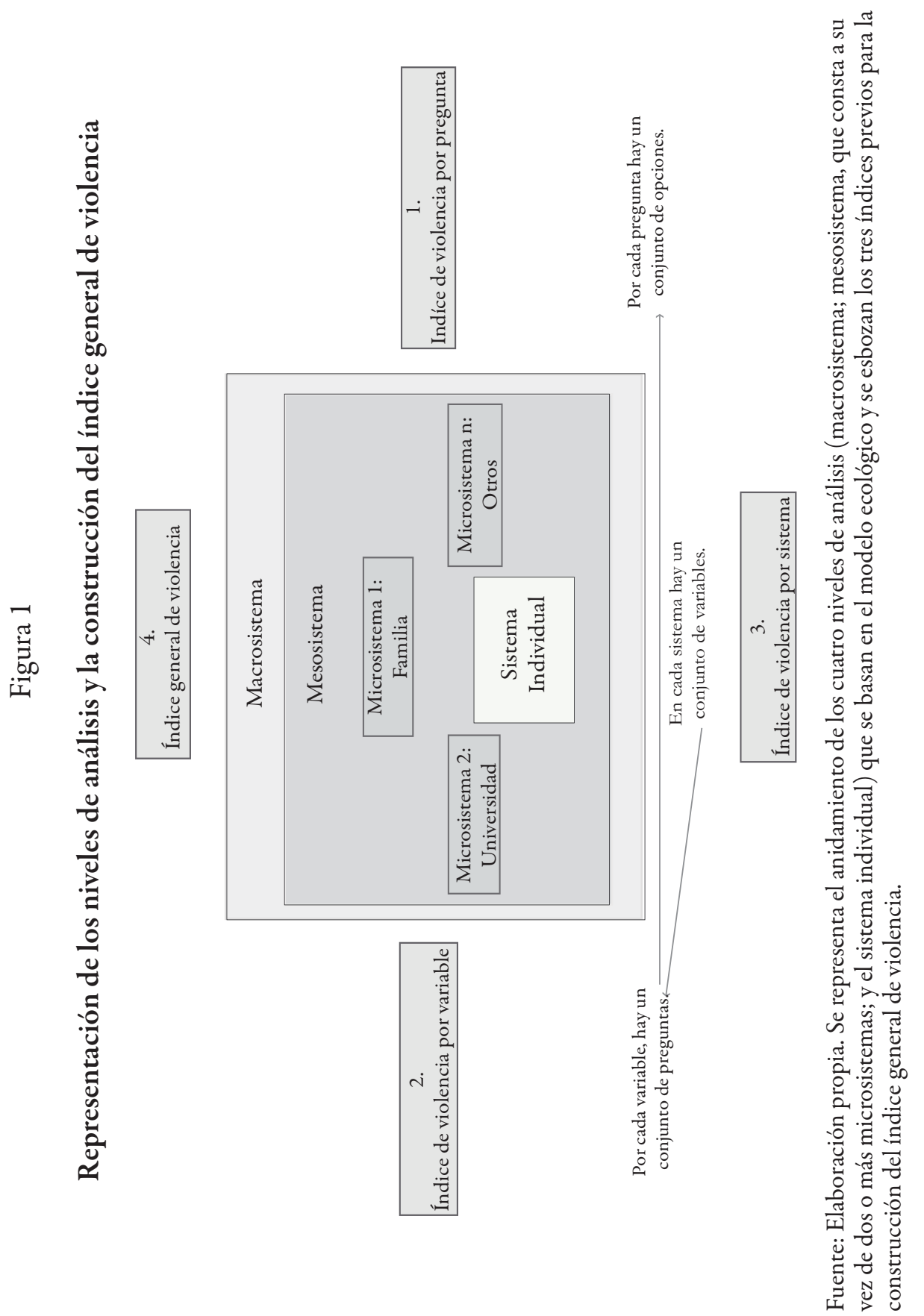
Francisco José Zamudio-Sánchez. Doctor en Filosofía, Universidad Estatal de Iowa. Profesor-Investigador de tiempo completo en la Universidad Autónoma Chapingo, México. Líneas de investigación: estadística social, análisis multivariado. Publicaciones recientes: "Dinámica de la calidad e inequidad del desarrollo humano en la región noreste de México, 19952005", en Estudios Fronterizos, vol. 14, núm. 27, México (2013); "Análisis sobre la evolución del desarrollo humano en la Península de Yucatán”, en Economía, Sociedad y Territorio, vol. XII, núm. 40, México: El Colegio Mexiquense (2012).

Marco Antonio Andrade-Barrera. Licenciado en Estadística, Universidad Autónoma Chapingo (UACh), México. Miembro del equipo de investigación en estadística social del Departamento de Estadística, Matemática y Cómputo de la UACh. Líneas de investigación: estadística social, ciencia de datos. Publicaciones recientes: Informe estadístico sobre desarrollo bumano en México 1995-2010, México: Universidad Autónoma Chapingo (2012); "Violencia de género en el alumnado de la Universidad Autónoma Chapingo”, Tesis de Licenciatura (2012).

Roxana Ivette Arana-Ovalle. Maestra en Ciencias (Estadística), Colegio de Postgraduados, México. Investigadora asociada en la UACh. Miembro del equipo de investigación en estadística social del Departamento de Estadística, Matemática y Cómputo de la UACh. Publicaciones recientes: "Una estrategia para portafolios con fondos cotizados", en Revista Española de Financiación y Contabilidad, vol. XLI, núm. 155, España: Asociación Española de Contabilidad y Administración de Empresas (2012); Estudio sobre la violencia de género: la otra cara de la Universidad Autónoma Chapingo, México: $\mathrm{UACh}$ (2013).

Arturo A. Alvarado-Segura. Doctor en Ciencias Biológicas por el Centro de Investigación Científica de Yucatán y estancia posdoctoral en la División de Ciencias Forestales de la Universidad Autónoma Chapingo (UACh). Docente en el Instituto Tecnológico Superior del Sur del Estado de Yucatán y colaborador del equipo de investigación en estadística social del Departamento de Estadística, Matemática y Cómputo de la UACh. Líneas de investigación: modelación en ciencias forestales, y la construcción y evaluación de índices de diversidad. Publicaciones recientes: "Factores que determinan el éxito o fracaso de proyectos forestales comunitarios con financiamiento gubernamental en México", en Bosque, vol. 36, Chile: Universidad Austral 
de Chile (2015); "Palm species richness, abundance and diversity in the Yucatan Peninsula, in a neotropical context", en Nordic Journal of Botany, vol. 30, Noruega: John Wiley \& Sons (2012); Análisis de diseños experimentales con igual número de submuestras, México: Universidad Autónoma Chapingo (1996).

Recepción: 17 de octubre de 2015.

Aprobación: 9 de febrero de 2017. 
